\title{
Syndecan-1 is overexpressed in human thoracic aneurysm but is dispensable for the disease progression in vivo
}

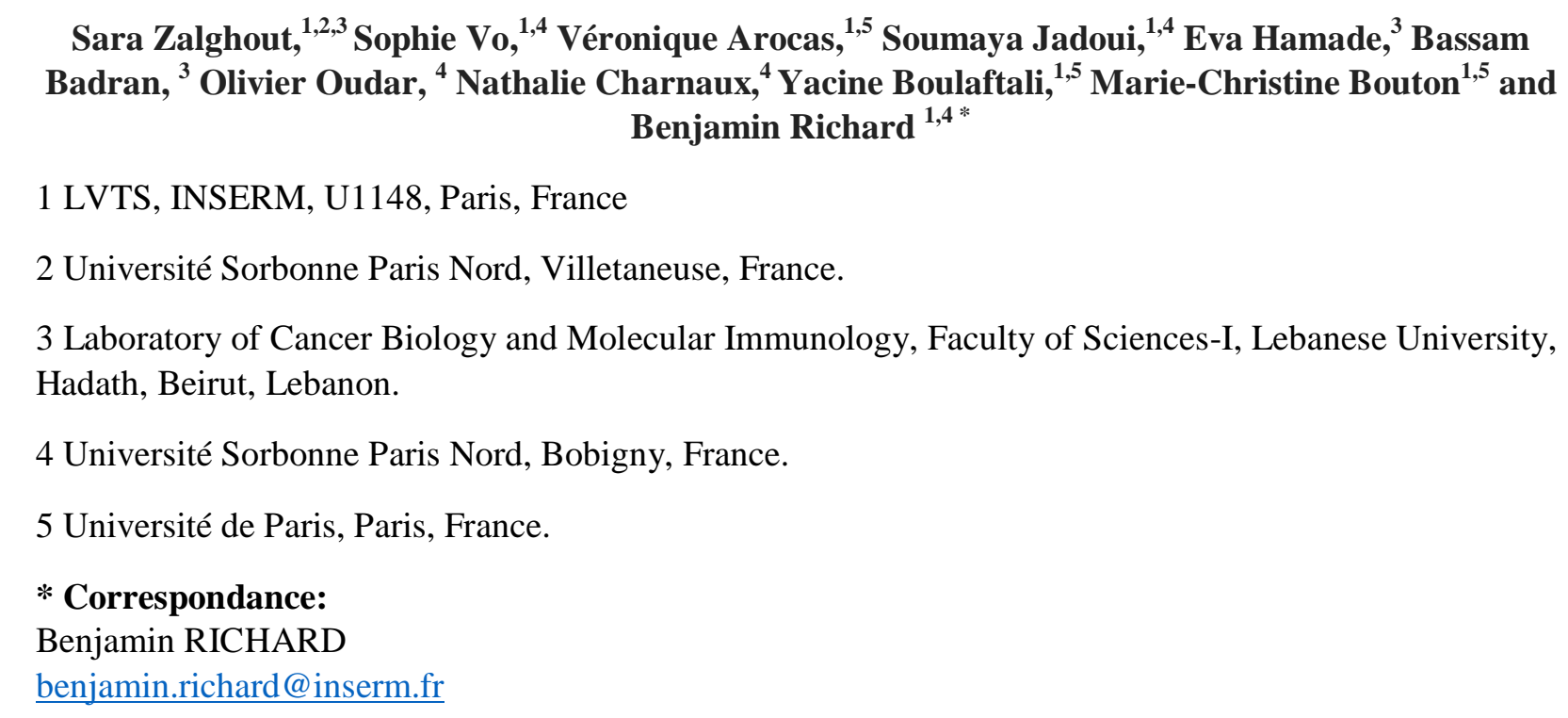

Keywords: Syndecan-1, aneurysm, proteoglycans, extracellular matrix, smooth muscle cell (SMC)

Number of words: 3667

Total number of figures and tables: 5 (excluding supplementary material) 
Zalghout et al.

\section{ABSTRACT}

21 Glycosaminoglycans (GAGs) pooling has been considered since long as one of the

22 histopathological characteristics defining thoracic aortic aneurysm (TAA) together with smooth

23 muscle cells (SMCs) apoptosis and elastin fibers degradation. However, few information is

24 provided about GAGs composition or potential implication in TAA pathology. Syndecan-1 (Sdc-

25 1) is a heparan sulfate proteoglycan that is implicated in extracellular matrix (ECM) interaction

26 and assembly, regulation of SMCs phenotype and various aspects of inflammation in the

27 vascular wall. In the current work, the regulation of Sdc-1 protein was examined in human TAA

28 by ELISA and immunohistochemistry. In addition, the role of Sdc-1 was evaluated in descending

29 TAA in vivo using a mouse model combining both aortic wall weakening and hypertension. Our

30 results showed that Sdc-1 protein is over expressed in human TAA aortas compared to healthy

31 counterparts and that SMCs are the major cell type expressing Sdc-1. Similarly, in the mouse

32 model used, Sdc-1 expression was increased in TAA aortas compared to healthy samples.

33 Although its protective role against abdominal aneurysm has been reported, we observed that

34 Sdc-1 was dispensable for TAA prevalence or rupture. In addition, Sdc-1 deficiency did not alter

35 the extent of aortic wall dilatation, elastin degradation, collagen deposition, or leukocyte

36 recruitment in our TAA model. These findings suggest that Sdc-1 could be a biomarker revealing

37 TAA pathology. Future investigations could uncover the underlying mechanisms leading to Sdc-

381 expression alteration in TAA. 
Zalghout et al.

\section{INTRODUCTION} 57 aortas $(10-12)$.

The mortality rate due to thoracic aortic aneurysm (TAA) rupture is in considerable increase and the efficiency of current drug therapies is yet limited and controversial (1-3). Glycosaminoglycans (GAGs) pooling has been considered since long as one of the histopathological characteristics defining TAAs, together with smooth muscle cells (SMCs) apoptosis or loss of contractility and elastin fibers degradation of (4-6). However, the identity of the accumulated GAGs in TAA and their potential involvement in the disease are poorly studied. A better understanding of the molecular changes occurring in TAA pathophysiology may open up new avenues for its treatment.

Even though proteoglycans (PGs; i.e. core protein with attached GAGs) occupy 1 to $5 \%$ mass fraction of a healthy arterial wall, they are crucial contributors to the aortic structural integrity and functioning and in particular to its mechanical homeostasis (7). PGs accumulation may exert a swelling pressure on the elastic laminae resulting in their separation and a consequent modification of the wall mechanical properties giving rise to a weakened wall and participating in TAA dissections and ruptures $(7,8)$. Recently, it has been reported that aggrecan and versican, two chondroitin sulfate PGs, accumulate in regions of medial degeneration in ascending TAAs and dissections and may contribute to extracellular matrix (ECM) disruption (9). In addition, the levels of heparan and chondroitin sulfate PGs are increased after vascular injury and in dissected

Syndecan-1 (Sdc-1), a transmembrane heparan sulfate PG, is involved in various aspects of inflammatory diseases (13-15), wound healing (16), and ECM interaction or assembly $(17,18)$. Sdc-1 has been shown to maintain a differentiated (19) and a contractile state of SMCs (12). In addition, it mediates cell-cell and cell-matrix interactions by acting as a co-receptor for binding 
Zalghout et al.

62 to ECM molecules or growth factors such as fibronectin or VEGF, respectively $(20,21)$.

63 Therefore, Sdc-1 is hypothesized to play a role in the development of aortic wall pathologies.

64 Sdc-1 has indeed been shown, in two mice models of abdominal aortic aneurysm (AAA) in

65 which aneurysm was induced by elastase or angiotensin II (Ang II) infusion (on Apo E deficient

66 background) (22), to exert a protective role against AAA development by attenuating the

67 inflammatory response and reducing protease activity (22). In contrast, no data are available

68 regarding its role in TAA development.

69 In the current study, we investigated Sdc-1 expression in the aortic wall of patients with TAA

70 arising from different etiologies. We also examined the disease progression and characteristics

71 by the use of an aneurysm mouse model generated by the combination of aortic wall weakness

72 and hypertension in Sdc- $1^{+/+}$or Sdc- $1^{-/-}$on C57B1/6J background.

MATERIALS AND METHODS

75

Human samples

77 Twenty-five human ascending TAA samples were obtained from patients at the time of

78 prophylactic surgical repair and eleven healthy thoracic aortic tissues were obtained from

79 anonymous deceased organ donors under the authorization of the French Biomedicine Agency

80 (PFS 09-007). Data relative to these patients are stated in Supplementary Table 1. Aortic

81 preparation involved direct paraformaldehyde fixation for histological studies or immediate

82 macroscopic dissection to separate the distinct aortic layers (intima, media, adventitia) followed

83 by direct freezing for q-PCR and ELISA analysis. 
Zalghout et al.

\section{Quantification of mRNA and protein levels of Sdc-1 in human samples}

85 Frozen media tissues from healthy or TAA aortas were pulverized using liquid nitrogen freezer 86 mill (6870 SPEX Certiprep 6750). Protein extraction was done by powder lysis in RIPA buffer

87 (5mg/ml). Sdc-1 media protein level was assessed using ELISA (R \& D systems, DY2780)

88 according to the manufacturer's instructions. For RNA extraction, 50 to $100 \mathrm{mg}$ of powder were

89 homogenized in Trizol Reagent (Invitrogen). Total RNA was extracted using RNeasy extraction

90 kit (Qiagen) and $1 \mu \mathrm{g}$ was reverse transcribed with RT Maxima First Strand kit (K1642, Thermo

91 Scientific). The q-PCR was performed on cDNA using Light Cycler (Roche). Levels of mRNA

92 were normalized to hypoxanthine guanine phosphoribosyl transferase (HPRT). Sequences of

93 primers are listed in table 1 . Fold changes of gene expression were calculated using $\Delta \Delta \mathrm{CT}$

94 method.

95

Table 1: Primers used for quantitative polymerase chain reaction (qPCR; human samples)

Gene $\quad$ Forward Primer Sequence (5'-3') Reverse Primer Sequence (3'-5')

HPRT1 AGTTGAGAGATCATCTCCAC TTGCTGACCTGCTGGATTAC

SDC1

TGTACCGCATGAAGAAGAAGG GTTtGGTGGGCTTCTGGTAG

97

$98 \quad$ Histology and immunostaining

99 For histological studies, human and murine fixed aortic tissues were embedded in paraffin, and

100 sectioned into $7 \mu \mathrm{m}$ thick sections. Antigen retrieval was done by heating the sections in a water

101 bath at $95^{\circ} \mathrm{C}$ for 30 min in a Dako Target Retrieval Solution, $\mathrm{pH} 9$ (code S236). Sections were 
Zalghout et al.

102

103

104

105

106

107

108

109

110

111

112

113

114

115

116

117 Elastin laminae degradation of mice aortas was blind-scored after orcein (Sigma-Aldrich)

permeabilized by $0.1 \%$ Triton-X 100 for $5 \mathrm{~min}$. For DAB IHC endogenous peroxidase activity was blocked by $3 \% \mathrm{H}_{2} \mathrm{O}_{2}$ for 30 min. Blocking was performed with $\mathrm{DAKO}{ }^{\circledR}$ protein blockserum free (code \# X0909) for $1 \mathrm{~h}$ at room temperature. Sections were incubated overnight at $4^{\circ} \mathrm{C}$ with primary antibodies (Supplementary Table 2).

For DAB IHC, slides were washed with PBS and treated $1 \mathrm{~h}$ at room temperature with LSAB2 System, HRP kit for human, and Peroxidase AffiniPure donkey anti-rat secondary antibody for mice samples (Supplementary Table 2). 3'-diaminobenzidine tetra-hydrochloride chromogen (DAB, K3468, Dako) was added to all sections and the reaction was stopped with distilled water. Counterstaining was done with hematoxylin (for mice IHC). Slides were then mounted with Eukitt ${ }^{\circ}$ mounting media.

For immunofluorescence, following primary antibody incubation, sections were treated with adequate secondary antibodies (Supplementary Table 2) for $2 \mathrm{~h}$ at room temperature. Nuclei were then stained with DAPI aqueous fluoroshield mounting media (Abcam, ab104139). $\alpha$ SMA, CD45 and ly6-G markers were used to identify SMCs, leukocytes and neutrophils, respectively.

118 staining, using an ascending scale from 0 to 4 (no degradation to maximal degradation). Collagen 119 deposition was detected with Sirius red (RAL Diagnostics) staining, and its quantification was 120 assessed by image $\mathrm{J}$ software after exposing the slides to polarized light (Leica microsystems, 121 DMi8).

\section{Animals and experimental procedures}


Zalghout et al.

123 Sdc- $1^{-/}$mice were a kind gift from Dr. Pyong Woo Park (Harvard Medical School, Boston) and

124 have been backcrossed for at least 10 generations on a C57BL/6J background.

125 Three week old Sdc- $1^{+/+}$and $\mathrm{Sdc}-1^{-/-}$mice were divided into three groups: (group 1) control 126 group with no treatment (mice were sacrificed at 8 weeks of age), (groups $2 \& 3$ ) treated groups

127 including mice receiving daily intraperitoneal injection of $150 \mathrm{mg} / \mathrm{kg} / \mathrm{day}$ of $\beta$ 128 aminopropionitrile fumarate (BAPN, A3134; Sigma Aldrich) for 28 days, then infused 129 subcutaneously with $1 \mu \mathrm{g} / \mathrm{kg} / \mathrm{min}$ of angiotensin II (Ang II, A9525, Sigma Aldrich) during 3 130 (group 2) or 28 days (group 3) using mini-osmotic pumps (Alzet 2004) (Figure 2A). At the end 131 of the protocol, mice were anesthetized and sacrificed. Aortas were harvested and rinsed with 132 saline to remove blood. Aortas were cleaned from the surrounding connective and adipose 133 tissues, fixed in $10 \%$ formalin for $24 \mathrm{~h}$ at $4^{\circ} \mathrm{C}$, and stored in $70 \%$ ethanol for further 134 investigations. Necropsy was done for mice that died during the course of the experiment and the 135 site of rupture (thoracic or abdominal) was determined based on macroscopic view of 136 hemorrhage location. Aneurysm (TAA or AAA) was defined as 50\% increase in the mean aortic 137 diameter compared to the same healthy aortic segment from non-treated mice (please check 138 below quantification section).

\section{Blood pressure measurement}

140 Mice blood pressure was measured non-invasively before pump implantation and up to 5 days 141 post-implantation, using the tail cuff system (BP-2000 SERIES II, Blood Pressure Analysis

142 System ${ }^{\mathrm{TM}}$, Visitech Systems). Mice were habituated for a minimum of 5 consecutive days 143 before the recording were considered.

\section{Measurement of aortic diameter}


Zalghout et al.

145 For morphometric analysis, images were taken by an EF-S $60 \mathrm{~mm}$ macro lens mounted on a 146 DSLR camera (Canon EOS600D), and used to measure the outer diameter of the early

147 descending thoracic aorta using Image $\mathrm{J}$ software. The diameter was determined at the site of 148 dilatation from the average of a minimum of 3 different measurements of the posterior and 149 inferior sides.

\section{Statistical analysis}

151 Values are shown as percentage or mean \pm standard error of the mean (SEM). Statistical analysis 152 was performed using Prism GraphPad. The non-parametric Mann-Whitney test was used to 153 compare two groups when data did not display a normal distribution. Fischer's exact test was 154 used when comparing two categorical variables. P values less than 0.05 were considered 155 significant.

157 RESULTS

158 Sdc-1 protein expression is increased in the aortic media of patients with TAA and is 159 expressed by SMCs

160 The expression of Sdc-1 at the mRNA and protein levels was investigated in human healthy or 161 TAA medial layer. Similar levels of SDC1 mRNA were observed between the different samples 162 (Figure 1A). In contrast, a significantly higher protein level of Sdc-1 was revealed by ELISA in 163 TAA compared with healthy human aortic media (Figure 1B). This difference was confirmed by 164 IHC (Figure 1C). Immunofluorescence results showed a co-localization between Sdc-1 and $\alpha$ 165 SMA, indicating that most of Sdc-1 is expressed by SMCs in human healthy and TAA aortas 166 (Figure 1D). 
Zalghout et al.

167

168

169

170

171

172

173

174

175

176

177

178

179

180

181

182

183

184

185

186

187

188

Altogether, these data illustrate that Sdc-1 is more expressed at the protein level in TAA aortas compared to healthy counterparts and SMCs are a type of cells overexpressing Sdc-1 in TAA aortas.

\section{Sdc-1 is overexpressed in the TAA developed in the BAPN/Ang II aneurysm mouse model}

The role of Sdc-1 on TAA development was investigated in an animal model using BAPN and Ang II known to induce aortic aneurysms in C57B1/6J mice (23). In the present model, 3-weeksold Sdc-1 $1^{+/+}$or Sdc- $1^{-/}$male mice received intraperitoneal injection of BAPN for 4 weeks and then subcutaneous infusion of Ang II by pump implantation. Mice received Ang II for 3 or 28 days to mimic early and late stages of TAA development (Figures 2A). The effect of Ang II on hypertension was confirmed by the significant increase in systolic blood pressure measured after implantation, with no difference between $\mathrm{Sdc}-1^{+/+}$and $\mathrm{Sdc}-1^{-/-}$mice (data not shown). The use of this Ang II/BAPN model did not generate aneurysm in all studied animals (discussed in the following section). Analysis of Sdc-1 expression by IHC, revealed that Sdc-1 protein was elevated in TAA compared to healthy aortas after both 3 and 28 days of Ang II treatment (Figure 2B). Sdc-1 expression was specific for the TAA development as Sdc-1 was not detected in aortas treated only with BAPN or with both BAPN and Ang II (at both time points) and that did not develop aneurysm (Supplementary Figure 1). Therefore, in accordance with human sample data, Sdc-1 expression is increased in the TAA generated in this mouse model.

Sdc-1 is dispensable for TAA development and displays a potential protective role against AAA development in mice 
Zalghout et al.

189 The survival rate of Sdc- $1^{+/+}$and Sdc- $1^{-/-}$mice was similar during the course of Ang II infusion 190 (Figures 3A), indicating that Sdc-1 deficiency did not alter the viability of the mice that 191 developed aneurysm.

192 As this model is known to induce both thoracic and abdominal aneurysms, TAA and AAA 193 incidence were compared in $\mathrm{Sdc}-1^{+/+}$and $\mathrm{Sdc}-1^{-/-}$mice. After 3 days of Ang II infusion, the 194 proportions of the developed aneurysms were as follow for $\mathrm{Sdc}-1^{+/+}$and $\mathrm{Sdc}-1^{-/-}$mice 195 respectively: TAA (40\% vs 35\%), AAA (20\% vs 6\%), both TAA and AAA (0\% vs 6\%) (Figure 196 3B). These results did not reveal any significant implication of Sdc-1 in aneurysm incidence in 197 this mouse model after 3 days of Ang II treatment.

198 When analyzing aneurysm incidence after 28 days of Ang II treatment, $73 \%$ of Sdc- $1^{+/+}$mice 199 developed TAA vs $52 \%$ in $\mathrm{Sdc}_{-1} 1^{-/}$mice. Moreover, $8 \%$ of $\mathrm{Sdc}-1^{-/-}$developed AAA whereas 200 none of Sdc-1+/+ mice did (Figure 3C). Sdc- ${ }^{-/-}$mice showed a higher tendency for AAA 201 occurrence, alone or in combination with TAA compared to Sdc- $1^{+/+}$mice, with $6 / 26$ AAA in $202 \mathrm{Sdc}^{-/-}$mice compared to 0/15 AAA in Sdc-1 ${ }^{+/+}$mice (Figure 3D). These data are in accordance 203 with a previous study reporting a protective effect of Sdc-1 against AAA development in a mice 204 model of AAA (22).

206 Sdc-1 deficiency does not alter the extent of aortic dilatation, ECM remodeling, or 207 leukocytes recruitment in descending TAA in mice

208 The observation that Sdc-1 was not involved in TAA incidence does not rule out the possibility 209 that it could affect the extent of thoracic aortic dilatation or the morphology of the developed 210 TAA. 
Zalghout et al.

211 Aortas harvested at 3 or 28 days from surviving animals were photographed (Figure 4A) and

212 their external diameters were measured. There was no difference in the descending thoracic

213 diameter between Sdc- $1^{+/+}$and $\mathrm{Sdc}-1^{-/-}$aortas at both time points (Figure 4B). More specifically,

214 we did not observe any difference in diameter between Sdc-1 ${ }^{+/+}$and Sdc- $1^{-/}$aortas that displayed

215 TAA, 28 days after pump implantation (data not shown).

216 The ECM remodeling of the developed Sdc-1 ${ }^{+/+}$or Sdc- $1^{-/-}$TAA was investigated by assessing

217 the level of elastic degradation and collagen deposition by orcein and Sirius red staining,

218 respectively. Similar levels of elastin degradation (Figure 4C) or collagen deposition (Figure 4D)

219 were observed for Sdc- $1^{+/+}$and $\mathrm{Sdc}-1^{-/-}$aortas with TAA.

220 Increasing evidence support a role of inflammation or immune cells infiltration in human TAA

221 (reviewed in (24)) and TAA mice models are often associated with inflammation (25,26). In

222 addition, Sdc-1 is involved in various aspects of inflammation such as leukocyte recruitment (27)

223 or its resolution (28). Therefore, leukocyte and more specifically neutrophil recruitment were

224 analyzed in $\mathrm{Sdc}-1^{+/+}$or Sdc- ${ }^{-/-}$TAA aortas after 3 (Supplementary Figure 2A) or 28 days

225 (Figure 4E, Supplementary Figure 2B) of Ang II infusion.

226 Massive recruitment of leukocytes (CD45 staining) and neutrophils (Ly-6G staining) in TAA

227 was observed in a similar manner in both $\mathrm{Sdc}-1^{+/+}$and $\mathrm{Sdc}-1^{-/-}$mice following 3 days of Ang II

228 infusion (Supplementary Figure 2A). Leukocytes and neutrophils were distributed nearly all over

229 the aorta but sparsely in the intact media. These cells were mainly observed in the adventitia or at

230 the border of the false channel and to a less extent in the dissected media part (media around

231 false channel) (Supplementary Figure 2A). Less leukocyte infiltration was detected after 28 days

232 of Ang II treatment compared to 3 days, with still no difference between $\mathrm{Sdc}-1^{+/+}$and $\mathrm{Sdc}-1^{-/-}$ 
Zalghout et al.

233 mice (Figure 4E, Supplementary Figure 2B). Moreover, very few neutrophils were observed in

234 the TAA formed in both Sdc-1 ${ }^{+/+}$and Sdc- $1^{-/-}$mice at this time point (Supplementary Figure 2B).

235 Taken together, these results indicate that Sdc-1 has no effect on the extent of aortic dilatation, 236 elastin degradation nor collagen deposition in descending TAA in mice. In addition, Sdc-1 does 237 not participate in leukocytes (and neutrophils) recruitment in descending TAA in this mouse 238 model.

\section{DISCUSSION}

240 Previous studies suggested that Sdc-1 could be an important player in TAA development as it is 241 involved in ECM assembly and organization (18), SMCs phenotype regulation and 242 mechanosensing (19). RNA level of Sdc-1 (referred previously as syndecan) was previously 243 reported to be increased after vascular injury (29). More recently, an increase of Sdc-1 protein

244 level was observed in the adventitia of human aortas with ascending TAA (30).

245 In the current study, we report for the first time, by ELISA and histological analysis, that Sdc-1

246 is one of the members of the PGs overexpressed in human TAA aorta medial layer. Our

247 immunofluorescence analysis showed that Sdc-1 expression is increased not only in media layer,

248 but also in the intima and adventitia layers, in agreement with Ntika et al (30).

249 Our results also indicate that SMCs are the major cell type expressing Sdc-1 in human TAA 250 aortas, which is in line with an in vitro study showing that mechanical stress induces the 251 expression of Sdc-1 by SMCs (31). This suggests that the altered wall mechanics in TAA aortas 252 induce Sdc-1 expression on SMCs.BAPN and Ang II infusion induce vascular remodeling (32), 253 TAA, AAA (23), and thoracic aortic dissection (33). Our model was adapted from a study 254 showing that BAPN and Ang II administration in C57B1/6J mice without any specific genetic 
Zalghout et al.

255

256

257

258

259

260

261

262

263

264

265

266

267

268

269

270

271

272

273

274

275

276

277

background, develop $49 \%$ of AAA and $38 \%$ of TAA mostly in the ascending part of the aorta (23). However, our Sdc- $1^{+/+} \mathrm{C} 57 \mathrm{~B} 1 / 6 \mathrm{~J}$ mice did not develop any AAA and almost all of the TAA were found in the descending aorta, at the typical site of B dissection and not distal as found by others (23). The observed differences between these results can be explained by the different age of the mice at the beginning of the experiment ( 8 vs. 3 weeks), the route of BAPN administration (subcutaneous vs. intraperitoneal), the duration of Ang II treatment (6 vs. 4 weeks), or the mouse background $\left(\mathrm{Sdcl}^{+/+}\right.$from heterozygous mating vs commercially available mice).

Our results indicate that Sdc-1 is not involved in the incidence of descending TAA neither in the risk of rupture in our model. However, Sdc-1 deficiency has been reported to exacerbate AAA formation and rupture vulnerability in two mice models of abdominal aneurysms (22). We observe similar findings as AAA was observed only in Sdc- $1^{-/-}$mice and not in the Sdc- $1^{+/+}$mice, 28 days after Ang II infusion. The fact that Sdc-1 was not involved in the extent of aortic dilatation, elastin degradation or collagen deposition in TAA in our model suggests that Sdc-1 may have a more potent effect on ECM remodeling in abdominal rather than in thoracic aorta given their different structural and mechanical characteristics $(34,35)$. The differences in the number of lamellar units, elastin and collagen content, proteinase system, and tension forces contribute to distinctive vascular remodeling at thoracic or abdominal sites (reviewed in (35)). Moreover, the inflammatory response is amplified in AAA compared to TAA (36), increasing the protease activity and MMPs production. It is worth to mention that PGs distribution is heterogeneous throughout the aorta providing a mechanism for regional dependent adaptation to variable hemodynamic stresses (37). More interestingly, the regulation of PGs has been shown to be distinct in the two types of the disease. For instance, aggrecan and versican accumulate in human ascending TAA (9), whereas proteomic analysis of AAA samples showed a reduced 
Zalghout et al.

278 abundance of these PGs in comparison to healthy samples (38). Therefore, it would not be

279 surprising that a single PG performs a distinct function specific to the site its of expression.

280

281

282

283

284

285

286

287

288

289

290

291

292

293

294

295

296

297

298

299

Notably, PGs display differential expression depending on the age of the individual (in mice or humans) and the severity of the disease (9,39-41). Examining the regulation of Sdc-1 in early human stages of TAA development is likely unachievable. We assumed that 3 days and 28 days of Ang II treatment corresponded to early and late stage of aneurysm, respectively. However, rupture (late stage of aneurysm) was observed all along the experimental protocol, even $24 \mathrm{~h}$ after Ang II infusion. Thus, the used model did not permit us to study a possible contribution of this PG in early stages of the disease.

As observed for the human TAA samples, Sdc-1 protein expression increased in mice TAA compared to healthy aortas at both time points. The observed over expression in the media and adventitial layers of TAA aortas 3 days after Ang II treatment should correspond to both SMC and leukocyte expression. Indeed 28 days after Ang II infusion, Sdc-1 overexpression was observed only in the media and not the adventitia of TAA aortas, in concordance with the observation of decreased leukocytes infiltration at this time point.

We observed an infiltration of neutrophils localized mainly in the adventitia, in borders of the false channel and in dissected media in TAA aortas 3 days after Ang II infusion most likely because Ang II as a vasopressor, is a potent stimulant of neutrophils recruitment $(42,43)$. Neutrophil infiltration was previously reported to be in intima following $24 \mathrm{~h}$ of Ang II infusion (44). Our data showed that after a longer time of Ang II infusion (3 days), which corresponds to a more advanced stage of aneurysm, neutrophil infiltration took place in the media where dissection was observed and in the adventitia. However, these neutrophils almost disappeared 28 
Zalghout et al.

300

301

302

303

304

305

306

307

308

309

310

311

\section{Conclusion}

313 This study reports an overexpression of Sdc-1 in human TAA compared to healthy aortas

days after Ang II treatment, corresponding possibly to a switch from an acute to a chronic inflammatory phase.

Despite the findings that neutrophil Sdc-1 reduces neutrophil adhesion to the endothelium (15) and that Sdc-1 mediates neutrophils resolution by chemokines clearance (28), our data showed that Sdc-1 does not play a role in neutrophil recruitment in our model, since no difference was observed between Sdc- $1^{+/+}$or Sdc- $1^{-/-}$TA aortas. More investigations are still required to identify the identity of PGs present in the distinct types of thoracic aneurysm. The impact of pooled PGs/GAGs on medial degeneration could be either a global effect generated by all accumulated PGs, or specific where each PG by itself exerts a particular role during TAA development. A process of compensation (or redundancy) displayed by other PG expression in the present model could explain the absence of Sdc-1 specific effect, as it has been reported in the context of atherosclerosis between biglycan and perlecan for Apo-B retention (45).

suggesting that it can serve as a biomarker for this pathology. The underlying mechanism relevant to this alteration remains to be determined. The mouse model used induced mostly descending TAA, and Sdc-1 was not involved in its incidence, neither in its histological characteristics. An implication of Sdc-1 in TAA (ascending or descending) could be revealed from studies in other TAA mouse models possibly associated with ECM genes deficiency as it organizes the ECM and interacts with its proteins. Interestingly, we observed that Sdc-1 tends to protect from AAA, in agreement with a previous report (22). Deciphering the protective 
Zalghout et al.

321 molecular function of Sdc-1 in AAA, and its possible role in TAA, could perhaps aid in the 322 comprehension of its clinical relevance.

\section{Ethics Statement}

325 Studies involving human participants were in agreement with the principles defined in the

326 Declaration of Helsinki. Written informed consents were obtained from all individual

327 participants (or family members) included in the study with Ethics Committee approval from

328 INSERM and AP-HP (CEERB du GHU Nord) Institutional review board (CPP 050432 ,

329 Ambroise Paré, Boulogne, France, April 2005; updated in March 2008). Mice experiments were

330 conducted according to the institutional guidelines for the care and use of animal research and

331 the ARRIVE guidelines and in compliance with the Animal Care and Use Committee (2011-

332 14/69-0035). The protocol was approved by the French MESRI (Ministère de l'Enseignement

333 Supérieur de la Recherche et de l'Innovation \# 8855)

334 Author Contributions

335 BR conceived and designed the study; SZ performed most of the experiments; BR, SV and SJ 336 performed some of human and/or mice experiments; MCB, VA, EH, YB, BR and SZ analysed

337 the data; SZ wrote the manuscript with support from BR; MCB, VA, YB, OO, NC, EH, and BB 338 supervised the research and provided intellectual discussion and editorial advice. All authors 339 contributed to manuscript revision and approved the submitted version.

\section{Conflict of Interest}


Zalghout et al.

341 The authors declare that the research was conducted in the absence of any commercial or

342 financial relationships that could be construed as a potential conflict of interest.

\section{Acknowledgments}

344 We thank Thierry Dubois and Benoit Ho-Tin-Noé for the critical reading of the manuscript and 345 helpful advices.

\section{$346 \quad$ Funding}

347 This work was funded by INSERM and Sorbonne Paris Nord University. Sara Zalghout was

348 funded by the Lebanese University, AZM Saade association, INSERM and Sorbonne Paris Nord 349 University. 
Zalghout et al.

1. Olsson Christian, Thelin Stefan, Ståhle Elisabeth, Ekbom Anders, Granath Fredrik. Thoracic Aortic Aneurysm and Dissection. Circulation (2006) 114:2611-2618. doi:10.1161/CIRCULATIONAHA.106.630400

2. Kuzmik GA, Sang AX, Elefteriades JA. Natural history of thoracic aortic aneurysms. Journal of Vascular Surgery (2012) 56:565-571. doi:10.1016/j.jvs.2012.04.053

3. Elefteriades JA, Botta DM. Indications for the Treatment of Thoracic Aortic Aneurysms. Surgical Clinics of North America (2009) 89:845-867. doi:10.1016/j.suc.2009.06.005

4. Shen Ying H., Lu Hong S., LeMaire Scott A., Daugherty Alan. Unfolding the Story of Proteoglycan Accumulation in Thoracic Aortic Aneurysm and Dissection. Arteriosclerosis, Thrombosis, and Vascular Biology (2019) 39:1899-1901. doi:10.1161/ATVBAHA.119.313279

5. Halushka MK, Angelini A, Bartoloni G, Basso C, Batoroeva L, Bruneval P, Buja LM, Butany J, d'Amati G, Fallon JT, et al. Consensus statement on surgical pathology of the aorta from the Society for Cardiovascular Pathology and the Association For European Cardiovascular Pathology: II. Noninflammatory degenerative diseases - nomenclature and diagnostic criteria. Cardiovascular Pathology (2016) 25:247-257. doi:10.1016/j.carpath.2016.03.002

6. Schlatmann TJM, Becker AE. Pathogenesis of dissecting aneurysm of aorta: Comparative histopathologic study of significance of medial changes. American Journal of Cardiology (1977) 39:21-26. doi:10.1016/S0002-9149(77)80005-2

7. Humphrey JD. Possible Mechanical Roles of Glycosaminoglycans in Thoracic Aortic Dissection and Associations with Dysregulated TGF- $\beta$. J Vasc Res (2013) 50:1-10. doi:10.1159/000342436

8. Roccabianca S, Ateshian GA, Humphrey JD. Biomechanical roles of medial pooling of glycosaminoglycans in thoracic aortic dissection. Biomech Model Mechanobiol (2014) 13:13-25. doi:10.1007/s10237-013-0482-3

9. Cikach FS, Koch CD, Mead TJ, Galatioto J, Willard BB, Emerton KB, Eagleton MJ, Blackstone EH, Ramirez F, Roselli EE, et al. Massive aggrecan and versican accumulation in thoracic aortic aneurysm and dissection. JCI Insight 3: doi:10.1172/jci.insight.97167

10. Cattell MA, Hasleton PS, Anderson JC. Glycosaminoglycan content is increased in dissecting aneurysms of human thoracic aorta. Clin Chim Acta (1994) 226:29-46. doi:10.1016/00098981(94)90100-7

11. Raines EW. The extracellular matrix can regulate vascular cell migration, proliferation, and survival: relationships to vascular disease. Int J Exp Pathol (2000) 81:173-182. doi:10.1046/j.13652613.2000.00155.x

12. Fukai N, Kenagy RD, Chen L, Gao L, Daum G, Clowes AW. Syndecan-1: An inhibitor of arterial smooth muscle cell growth and intimal hyperplasia. Arterioscler Thromb Vasc Biol (2009) 29:13561362. doi:10.1161/ATVBAHA.109.190132

13. Götte M, Joussen AM, Klein C, Andre P, Wagner DD, Hinkes MT, Kirchhof B, Adamis AP, Bernfield M. Role of Syndecan-1 in Leukocyte-Endothelial Interactions in the Ocular Vasculature. Invest Ophthalmol Vis Sci (2002) 43:1135-1141. 
Zalghout et al.

14. Pei S, Zheng D, Wang Z, Hu X, Pan S, Wang H. Elevated soluble syndecan-1 levels in neuromyelitis optica are associated with disease severity. Cytokine (2018) 111:140-145. doi:10.1016/j.cyto.2018.08.017

15. Masouleh BK, Dam GBT, Wild MK, Seelige R, Vlag J van der, Rops AL, Echtermeyer FG, Vestweber D, Kuppevelt TH van, Kiesel L, et al. Role of the Heparan Sulfate Proteoglycan Syndecan-1 (CD138) in Delayed-Type Hypersensitivity. The Journal of Immunology (2009) 182:4985-4993. doi:10.4049/jimmunol.0800574

16. Stepp MA, Gibson HE, Gala PH, Iglesia DDS, Pajoohesh-Ganji A, Pal-Ghosh S, Brown M, Aquino $\mathrm{C}$, Schwartz AM, Goldberger O, et al. Defects in keratinocyte activation during wound healing in the syndecan-1-deficient mouse. Journal of Cell Science (2002) 115:4517-4531. doi:10.1242/jcs.00128

17. Vanhoutte Davy, Schellings Mark W.M., Götte Martin, Swinnen Melissa, Herias Veronica, Wild Martin K., Vestweber Dietmar, Chorianopoulos Emmanuel, Cortés Víctor, Rigotti Attilio, et al. Increased Expression of Syndecan-1 Protects Against Cardiac Dilatation and Dysfunction After Myocardial Infarction. Circulation (2007) 115:475-482. doi:10.1161/CIRCULATIONAHA.106.644609

18. Xian X, Gopal S, Couchman J. Syndecans as receptors and organizers of the extracellular matrix. Cell and tissue research (2009) 339:31-46. doi:10.1007/s00441-009-0829-3

19. Chaterji S, Lam CH, Ho DS, Proske DC, Baker AB. Syndecan-1 regulates vascular smooth muscle cell phenotype. PLoS One (2014) 9:e89824. doi:10.1371/journal.pone.0089824

20. Alexopoulou AN, Multhaupt HAB, Couchman JR. Syndecans in wound healing, inflammation and vascular biology. Int J Biochem Cell Biol (2007) 39:505-528. doi:10.1016/j.biocel.2006.10.014

21. Rhodes JM, Simons M. The extracellular matrix and blood vessel formation: not just a scaffold. $J$ Cell Mol Med (2007) 11:176-205. doi:10.1111/j.1582-4934.2007.00031.x

22. Xiao J, Angsana J, Wen J, Smith SV, Park PW, Ford ML, Haller CA, Chaikof EL. Syndecan-1 Displays a Protective Role in Aortic Aneurysm Formation by Modulating T Cell-Mediated Responses. Arterioscler Thromb Vasc Biol (2012) 32:386-396. doi:10.1161/ATVBAHA.111.242198

23. Kanematsu Y, Kanematsu M, Kurihara C, Tsou T-L, Nuki Y, Liang EI, Makino H, Hashimoto T. Pharmacologically Induced Thoracic and Abdominal Aortic Aneurysms in Mice. Hypertension (2010) 55:1267-1274. doi:10.1161/HYPERTENSIONAHA.109.140558

24. Pisano C, Balistreri CR, Ricasoli A, Ruvolo G. Cardiovascular Disease in Ageing: An Overview on Thoracic Aortic Aneurysm as an Emerging Inflammatory Disease. Mediators Inflamm (2017) 2017:1274034. doi:10.1155/2017/1274034

25. Dinesh NEH, Reinhardt DP. Inflammation in thoracic aortic aneurysms. Herz (2019) 44:138-146. doi:10.1007/s00059-019-4786-7

26. Malecki C, Hambly BD, Jeremy RW, Robertson EN. The Role of Inflammation and Myeloperoxidase-Related Oxidative Stress in the Pathogenesis of Genetically Triggered Thoracic Aortic Aneurysms. Int J Mol Sci (2020) 21:7678. doi:10.3390/ijms21207678 
Zalghout et al.

27. Angsana J, Chen J, Smith S, Xiao J, Wen J, Liu L, Haller CA, Chaikof EL. Syndecan-1 Modulates the Motility and Resolution Responses of Macrophages. Arteriosclerosis, Thrombosis, and Vascular Biology (2015) 35:332-340. doi:10.1161/ATVBAHA.114.304720

28. Hayashida K, Parks WC, Park PW. Syndecan-1 shedding facilitates the resolution of neutrophilic inflammation by removing sequestered CXC chemokines. Blood (2009) 114:3033-3043. doi:10.1182/blood-2009-02-204966

29. Nikkari ST, Järveläinen HT, Wight TN, Ferguson M, Clowes AW. Smooth muscle cell expression of extracellular matrix genes after arterial injury. Am J Pathol (1994) 144:1348-1356.

30. Ntika S, Tracy LM, Franco-Cereceda A, Björck HM, Krizhanovskii C. Syndecan-1 Expression Is Increased in the Aortic Wall of Patients with Type 2 Diabetes but Is Unrelated to Elevated Fasting Plasma Glucagon-Like Peptide-1. Biomedicines (2021) 9:697. doi:10.3390/biomedicines9060697

31. Julien MA, Haller CA, Wang P, Wen J, Chaikof EL. Mechanical strain induces a persistent upregulation of syndecan-1 expression in smooth muscle cells. J Cell Physiol (2007) 211:167-173. doi:10.1002/jcp.20927

32. Eberson LS, Sanchez PA, Majeed BA, Tawinwung S, Secomb TW, Larson DF. Effect of Lysyl Oxidase Inhibition on Angiotensin II-Induced Arterial Hypertension, Remodeling, and Stiffness. PLOS ONE (2015) 10:e0124013. doi:10.1371/journal.pone.0124013

33. Ren W, Liu Y, Wang X, Jia L, Piao C, Lan F, Du J. $\beta$-Aminopropionitrile monofumarate induces thoracic aortic dissection in C57BL/6 mice. Sci Rep (2016) 6: doi:10.1038/srep28149

34. Jana S, Hu M, Shen M, Kassiri Z. Extracellular matrix, regional heterogeneity of the aorta, and aortic aneurysm. Exp Mol Med (2019) 51:1-15. doi:10.1038/s12276-019-0286-3

35. Ruddy JM, Jones JA, Spinale FG, Ikonomidis JS. Regional Heterogeneity within the Aorta: Relevance to Aneurysm Disease. J Thorac Cardiovasc Surg (2008) 136:1123-1130. doi:10.1016/j.jtcvs.2008.06.027

36. Tang PCY, Yakimov AO, Teesdale MA, Coady MA, Dardik A, Elefteriades JA, Tellides G. Transmural inflammation by interferon-gamma-producing $\mathrm{T}$ cells correlates with outward vascular remodeling and intimal expansion of ascending thoracic aortic aneurysms. FASEB J (2005) 19:1528-1530. doi:10.1096/fj.05-3671fje

37. Azeloglu EU, Albro MB, Thimmappa VA, Ateshian GA, Costa KD. Heterogeneous transmural proteoglycan distribution provides a mechanism for regulating residual stresses in the aorta. American Journal of Physiology-Heart and Circulatory Physiology (2008) 294:H1197-H1205. doi:10.1152/ajpheart.01027.2007

38. Didangelos A, Yin X, Mandal K, Saje A, Smith A, Xu Q, Jahangiri M, Mayr M. Extracellular Matrix Composition and Remodeling in Human Abdominal Aortic Aneurysms: A Proteomics Approach. Mol Cell Proteomics (2011) 10:M111.008128. doi:10.1074/mcp.M111.008128

39. Wen J, Wang P, Smith SV, Haller CA, Chaikof EL. Syndecans are differentially expressed during the course of aortic aneurysm formation. Journal of Vascular Surgery (2007) 46:1014-1025. doi:10.1016/j.jvs.2007.06.022 
Zalghout et al.

40. Tovar AMF, Cesar DCF, Leta GC, Mourão PAS. Age-Related Changes in Populations of Aortic Glycosaminoglycans: Species With Low Affinity for Plasma Low-Density Lipoproteins, and Not Species With High Affinity, Are Preferentially Affected. ATVB (1998) 18:604-614. doi:10.1161/01.ATV.18.4.604

41. Yasmin, Maskari RA, McEniery CM, Cleary SE, Li Y, Siew K, Figg NL, Khir AW, Cockcroft JR, Wilkinson IB, et al. The matrix proteins aggrecan and fibulin-1 play a key role in determining aortic stiffness. Sci Rep (2018) 8:8550. doi:10.1038/s41598-018-25851-5

42. Nabah YNA, Losada M, Estellés R, Mateo T, Company C, Piqueras L, Lopez-Gines C, Sarau H, Cortijo J, Morcillo EJ, et al. CXCR2 Blockade Impairs Angiotensin II-Induced CC Chemokine Synthesis and Mononuclear Leukocyte Infiltration. Arteriosclerosis, Thrombosis, and Vascular Biology (2007) 27:2370-2376. doi:10.1161/ATVBAHA.107.147009

43. Arndt PG, Young SK, Poch KR, Nick JA, Falk S, Schrier RW, Worthen GS. Systemic Inhibition of the Angiotensin-Converting Enzyme Limits Lipopolysaccharide-Induced Lung Neutrophil Recruitment through Both Bradykinin and Angiotensin II-Regulated Pathways. The Journal of Immunology (2006) 177:7233-7241. doi:10.4049/jimmunol.177.10.7233

44. Kurihara Tomohiro, Shimizu-Hirota Ryoko, Shimoda Masayuki, Adachi Takeshi, Shimizu Hideyuki, Weiss Stephen J., Itoh Hiroshi, Hori Shingo, Aikawa Naoki, Okada Yasunori. Neutrophil-Derived Matrix Metalloproteinase 9 Triggers Acute Aortic Dissection. Circulation (2012) 126:3070-3080. doi:10.1161/CIRCULATIONAHA.112.097097

45. Tang T, Thompson JC, Wilson PG, Yoder MH, Müeller J, Fischer JW, Jon Williams K, Tannock LR. Biglycan deficiency: increased aortic aneurysm formation and lack of atheroprotection. $J \mathrm{Mol}$ Cell Cardiol (2014) 75:174-180. doi:10.1016/j.yjmcc.2014.07.014 
Zalghout et al.

A

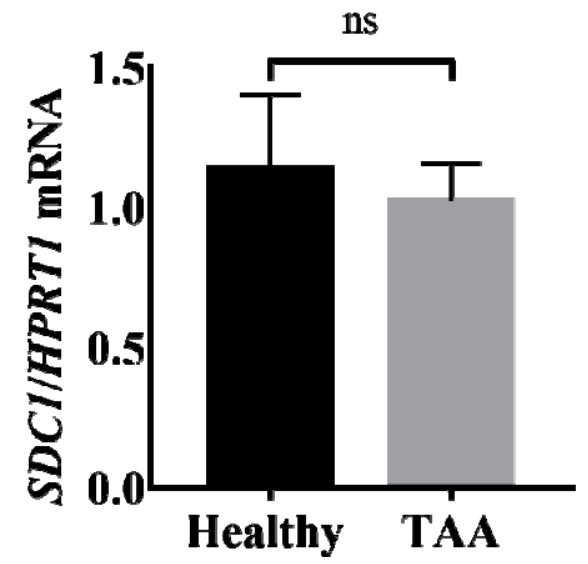

C

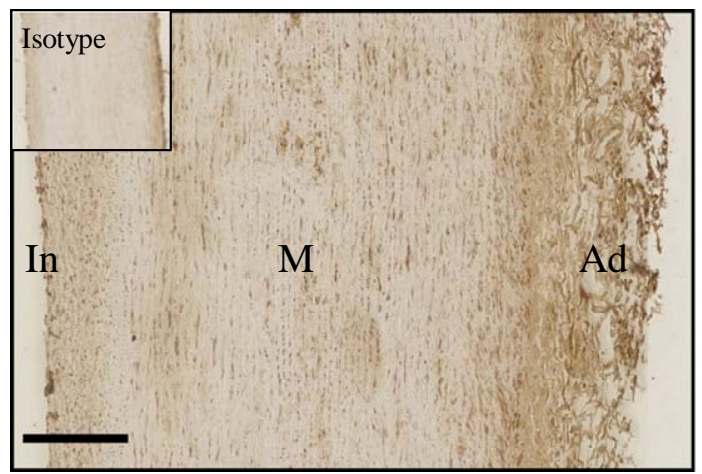

D
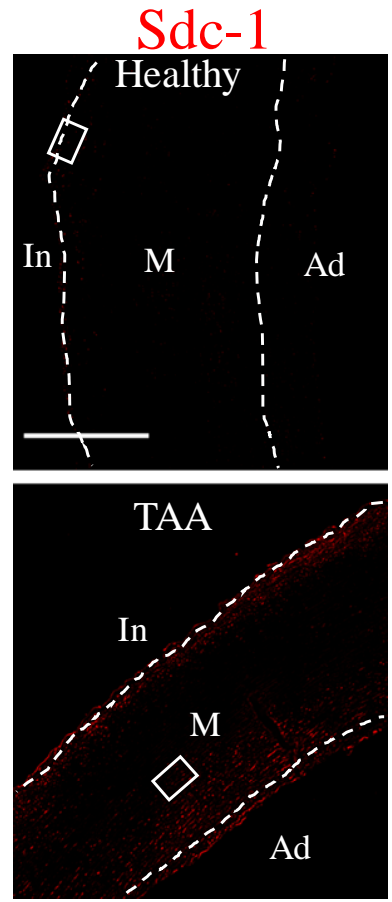

B
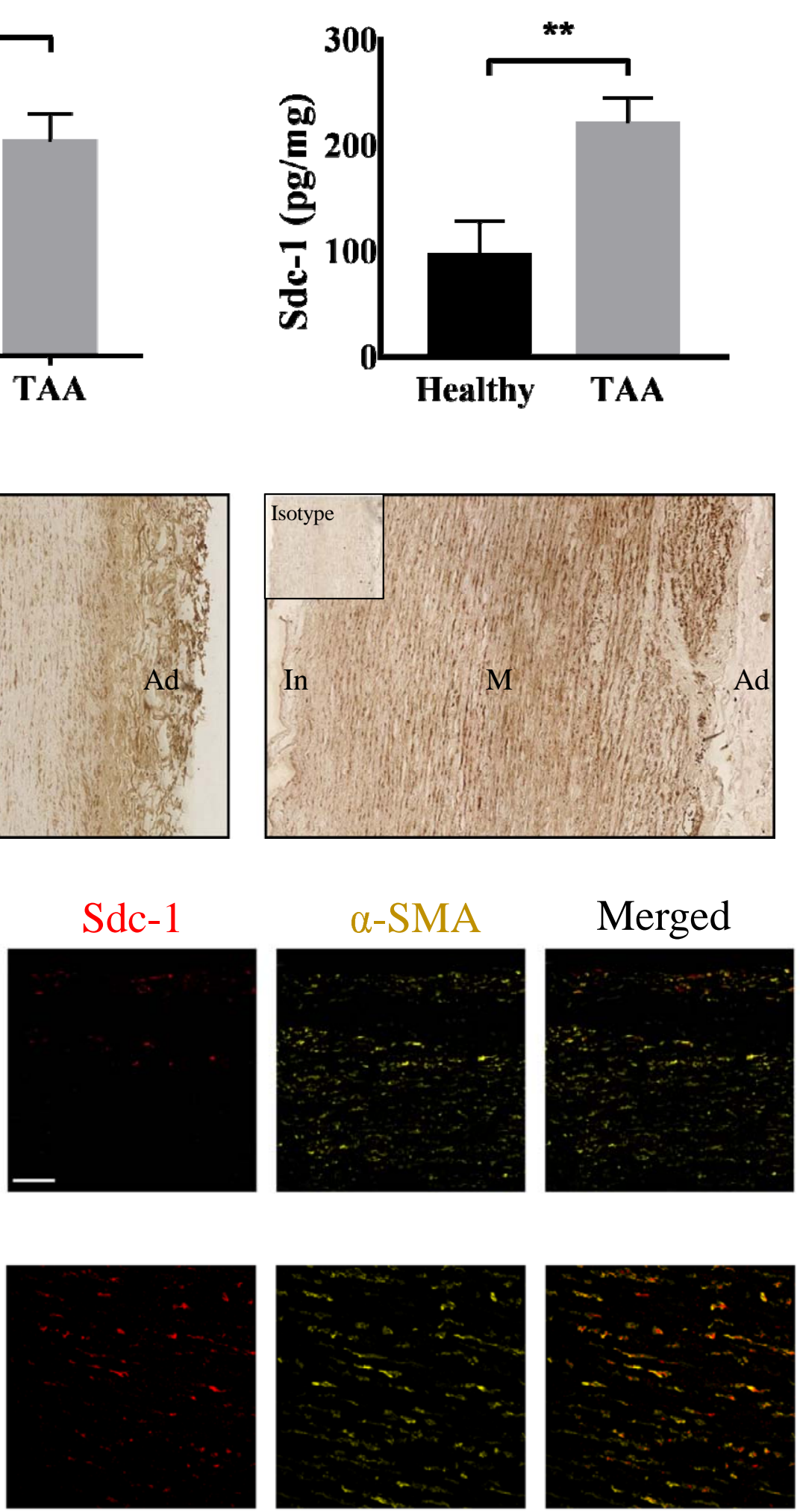

Figure 1 
Zalghout et al.

Figure 1. Sdc-1 protein level is increased in human TAA media compared to healthy ones

\section{and is expressed by SMCs}

(A) mRNA level of SDCl was measured by q-PCR and normalized to Hypoxanthine-guanine phosphoribosyltransferase (HPRT) in healthy $(n=6)$ and TAA $(n=14)$ human thoracic aortic media. (B) The concentration of Sdc-1 protein in human aortic media was assessed by ELISA

503 from healthy $(n=8)$ or TAA $(n=16)$ donors. (C) Representative images of Sdc-1 staining by 504 immunohistochemistry in human healthy and TAA aortas. Staining with an isotype control was 505 performed as a negative control and shown on the top left of images. Scale bar corresponds to $506200 \mu \mathrm{m}$. (D) Representative immunofluorescence images of Sdc-1 (red) and $\alpha$-SMA (yellow) co507 staining in human healthy (top images) or TAA (bottom images) aortas. The dashed lines 508 indicate the separation of the different layers: In: intima, M: media, Ad: adventitia. Scale bar 509 corresponds to $400 \mu \mathrm{m}$ for the non-magnified images and $50 \mu \mathrm{m}$ to the magnified ones. (A, B) 510 Data are presented as mean \pm SEM and $P$ values were calculated using two-tailed Mann-Whitney 511 test; $* *$ corresponds to $p$ value $<0.01$, ns: not significant. 
Zalghout et al.

A
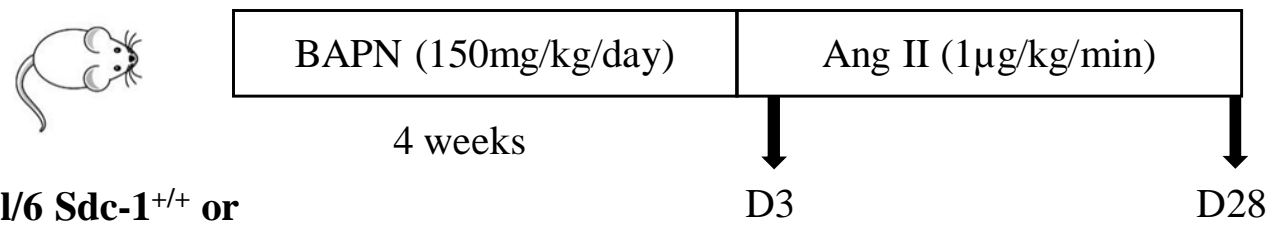

C57Bl/6 Sdc- $1^{+/+}$or

Sdc-1/-

$\hat{3}$ weeks

B

Sdc- $1^{+/+}$healthy

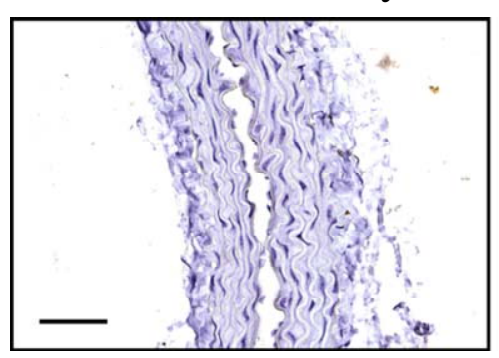

Sdc- $1^{+/+}$TAA

3D Ang II

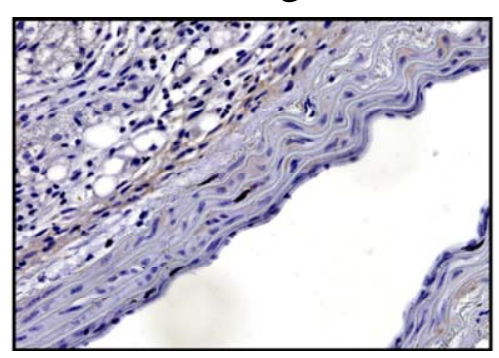

28D Ang II

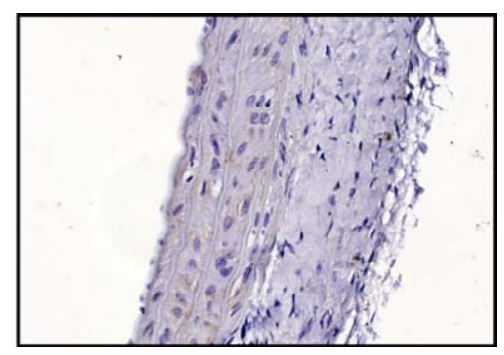

\section{Figure 2}


Zalghout et al.

512 Figure 2. Sdc-1 is overexpressed in TAA compared to healthy aortas in the BAPN/AngII aneurysm

513 mouse model

514 (A) Experimental model used. Sdc- $1^{+/+}$or Sdc- $1^{-/-}$male C57B1/6J mice of 3 weeks old received

515 intraperitoneal injection of $\beta$-amino propionitrile (BAPN) for 4 weeks followed by subcutaneous

516 infusion of angiotensin II (Ang II) by pump implantation for 3 (D3) or 28 (D28) days, then

517 sacrificed. (B) Representative images of Sdc-1 immunostaining in healthy $(n=3)$ and TAA aortas

518 after $3(n=2)$ or 28 days $(n=3)$ of Ang II treatment. Scale bar corresponds to $50 \mu \mathrm{m}$. 
Zalghout et al.

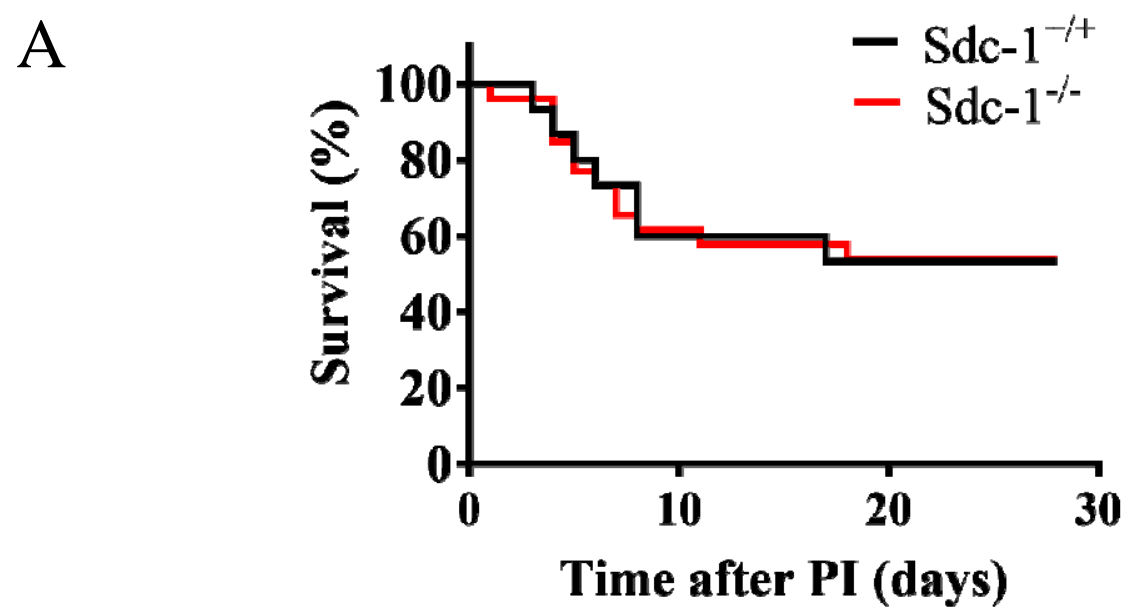

B
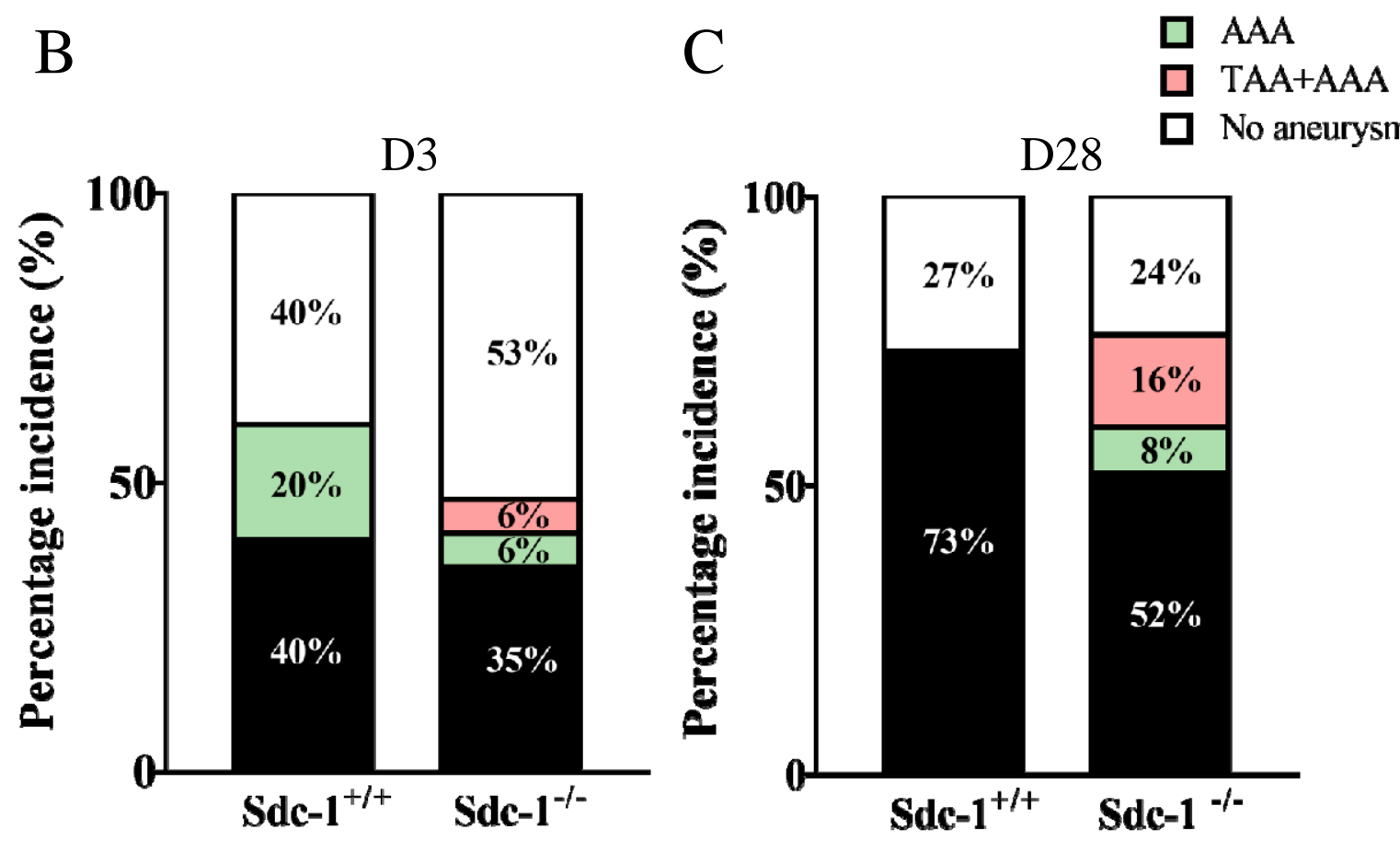

D

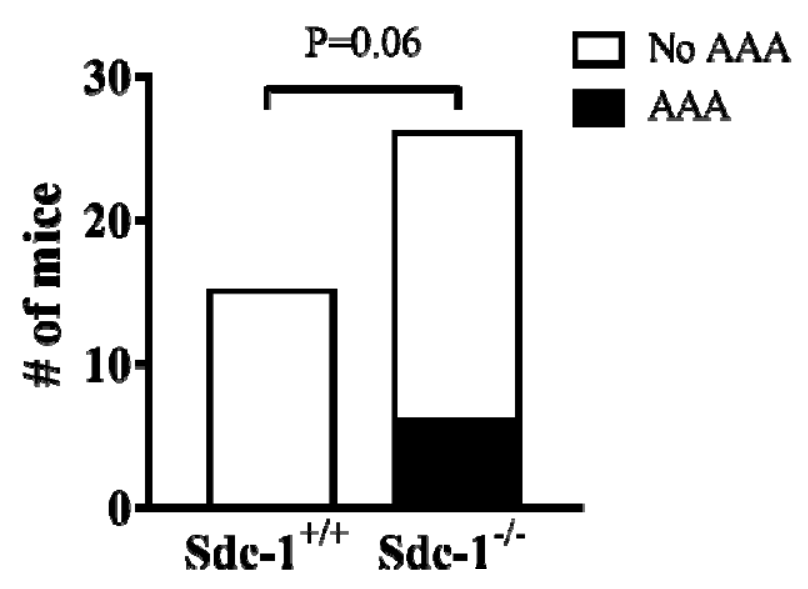

Figure 3 
Zalghout et al.

519 Figure 3. Sdc-1 is dispensable for TAA incidence or rupture but tends to protect from AAA 520 development in mice

521 (A) Survival rate of mice after 28 days following Ang II infusion Gehan-Breslow-Wilcoxon test.

522 PI : pump implantation. Sdc- $1^{+/+}: n=15, \mathrm{Sdc}^{-1^{--}}: n=26$. ns, non-significant. (B, C) Percentage

523 of TAA or AAA incidence in $\mathrm{Sdc}-1^{+/+}$or $\mathrm{Sdc}-1^{-/-}$mice for 3 (B) or 28 days (C) of Ang II

524 treatment. (D) AAA incidence in mice treated with Ang II for 28 days. Fisher's exact test. (B)

$525 \operatorname{Sdc}^{+/+}: n=5, \mathrm{Sdc}^{-1^{-/}}: n=17 .(\mathbf{C}, \mathbf{D}) \mathrm{Sdc}-1^{+/+}: n=15, \mathrm{Sdc}-1^{-/-}: n=26$. 
Zalghout et al.

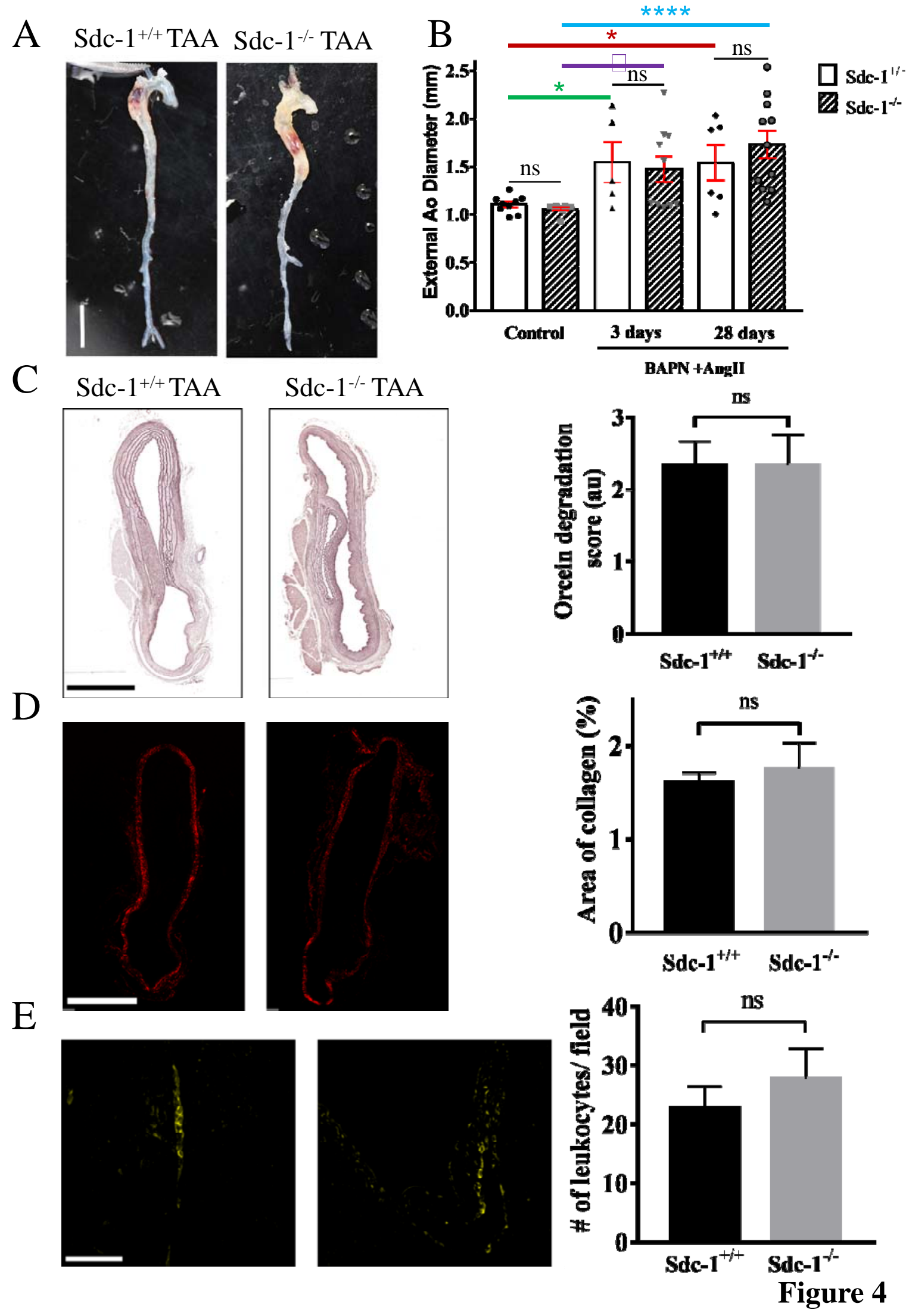


Zalghout et al.

\section{Figure 4. Sdc-1 deficiency does not alter the extent of aortic dilatation, ECM remodeling,} or leukocyte recruitment in descending TAA in mice

(A) Macroscopic images of developed TAA in Sdc- $1^{+/+}$or Sdc- $1^{-/-}$mice. Scale bar corresponds to $5 \mathrm{~mm}$. (B) Measurement of external descending thoracic diameter in Sdc- $1^{+/+}$and Sdc- $1^{-/-}$mice that received BAPN and Ang II for 3 or 28 days, and control (sham) mice. Aorta with a diameter $\geq 1.5 \mathrm{~mm}$ was considered as an aorta that developed TAA. Sdc- $1^{+/+}$or Sdc- $1^{-/-} \operatorname{ctrl}: n=9$ for both, Sdc- $1^{+/+}$and Sdc- $1^{-/-} 3$ days: $n=5$ and $n=10$ respectively, Sdc- $1^{+/+}$and Sdc- $1^{-/-} 28$ days: $n=6$ and $n=11$ respectively. (C) Elastin degradation. Representative images of orcein staining for Sdc- $1^{+/+}$ or Sdc- $1^{-/-}$TAA aortas after 28 days following Ang II treatment. Histological sections were evaluated for elastin degradation by giving an approximate score (scale from 0 to $4: 0$ corresponding to no degradation and 4 to maximal degradation). Sdc- $1^{+/+}: n=3$, Sdc- $1^{-/}: n=6$ (D) Collagen deposition. Representative images of Sirius red staining (visualized under polarized light) and its quantification by image $\mathrm{J}$ software. $P$ values were calculated using two-tailed Mann-Whitney test. (C, D) Scale bar corresponds to $500 \mu \mathrm{m}$. (E) Representative images of immunofluorescence staining of leukocytes (CD45) in TAA aortas after 28 days of Ang II infusion and its quantification (to the right) by Image J software. Scale bar corresponds to $50 \mu \mathrm{m}$. (D, E) Sdc- $1^{+/+}: n=3, \operatorname{Sdc}-1^{-/-}: n=7 .(\mathbf{B}, \mathbf{C}, \mathbf{D}, \mathbf{E})$ Data are presented as mean $\pm \mathrm{SEM}$ and $P$ values were calculated using two-tailed Mann-Whitney test; *: p<0.05, $\square: \mathrm{p}<0.01$, ****: $\mathrm{p}<0.0001, \mathrm{~ns}$ : not significant. 